\title{
Philosophiques
}

\section{Wittgenstein, Kripke et le paradoxe des règles (II)}

\section{Denis Sauvé}

Volume 21, numéro 1, printemps 1994

URI : https://id.erudit.org/iderudit/027254ar

DOI : https://doi.org/10.7202/027254ar

Aller au sommaire du numéro

Éditeur(s)

Société de philosophie du Québec

ISSN

0316-2923 (imprimé)

1492-1391 (numérique)

Découvrir la revue

Citer cet article

Sauvé, D. (1994). Wittgenstein, Kripke et le paradoxe des règles (II). Philosophiques, 21(1), 137-157. https://doi.org/10.7202/027254ar

\section{Résumé de l'article}

L'exposé du scepticisme sémantique par Kripke dans son ouvrage intitulé Wittgenstein on Rules and Private Language est généralement considéré comme intéressant et philosophiquement stimulant mais non comme une exégèse plausible des vues de Wittgenstein dans ses Recherches philosophiques. Cet article a pour but de montrer au contraire que l'interprétation de Kripke donne un exposé correct de certaines (au moins) des vues les plus importantes de Wittgenstein sur le concept de suivre une règle dans les Recherches. 


\title{
WIITGENSIEN, KRIPKE ET LE PARADOXE DES RÈGIES (II)
}

\author{
par \\ Denis Sauvé
}

\begin{abstract}
RÉSUMÉ : L'exposé du scepticisme sémantique par Kripke dans son ouvrage intitulé Wittgenstein on Rules and Private Language est généralement considéré comme intéressant et philosophiquement stimulant mais non comme une exégèse plausible des vues de Wittgenstein dans ses Recherches philosophiques. Cet article a pour but de montrer au contraire que l'interprétation de Kripke donne un exposé correct de certaines (au moins) des vues les plus importantes de Wittgenstein sur le concept de suivre une règle dans les Recherches.
\end{abstract}

\begin{abstract}
Kripke's exposition of semantic scepticism in Wittgenstein on Rules and Private Language is widely regarded as an interesting and stimulating piece of philosophical argumentation though not as a plausible exegesis of Wittgenstein's own views in the Philosophical Investigations. The aim of this paper is to show on the contrary that Kripke gives a correct reading of at least some of Wittgenstein's most important views on rule-following in the Investigations.
\end{abstract}

Kripke avance dans Wittgenstein on Rules and Private Language l'interprétation d'après laquelle Wittgenstein défend une forme de «scepticisme sémantique », c'est-à-dire le point de vue consistant à soutenir qu'« lill ne peut rien y avoir de tel que signifier quoi que ce soit par un mot las meaning anything by any wordl ${ }^{\mathrm{I}}$. » J'ai essayé de montrer dans la première partie de cette étude ${ }^{2}$ qu'il découle du « paradoxe des règles » de Wittgenstein que personne ne peut suivre une règle correctement (n'importe quelle action, dit-il, peut être mise en accord avec une

I. S. Kripke, Wittgenstein on Rules and Private Language, Oxford, Basil Blackwell, 1982, p. 55. Les références à cet ouvrage seront signalées par l'abréviation «WRPL ».

2. «Wittgenstein, Kripke et le paradoxe des régles (I) », Philosophiques, vol. XX, p. 2546. 
règle suivant une interprétation ou une autre de la règle) et, si on se réfère en particulier aux règles de langage, la conséquence en est qu'il n'y a rien de tel qu'employer un mot correctement - pour « signifier » quelque chose. Kripke utilise l'exemple du mot «plus » (ou du concept d'addition) : si « $R_{I} »$ est la règle habituelle pour effectuer des additions, on peut concevoir une autre règle $(R 2)$, de « second niveau », gouvernant l'application de la première, qui serait par exemple : «Si les nombres $x$ et $y$ sont plus petits que ou égaux à 57 , appliquer RI; dans tous les autres cas, écrire " 5 " » (de sorte que, si quelqu'un qui suit $R 2$ se demandait quelle est la somme de 68 et de 57 , il devrait répondre « 5 » et non «25 »). Une autre règle (de « troisième niveau ») pourrait être : « Appliquer $R 2$ seulement à partir de l'an trois mille; avant l'an trois mille appliquer Rı. »Wittgenstein cite dans les Recherches philosophiques l'exemple analogue d'une règle pour former des suites de nombres (la règle « Pour tout $n$, écrire $\left.n+2 ! »\left[P U{ }_{185}\right]\right)^{3}$. Celle-ci pourrait être « interprétée » (ou « expliquée ») à l'aide d'une autre règle (de second niveau), et cette dernière également en recourant à une troisième règle et ainsi du suite (cf. PU 86 et $W L C$ p. I32-133). Si toute règle peut être interprétée ou expliquée de plusieurs façons différentes, la conclusion qui s'ensuit est qu'il n'y a pas d'application correcte d'une règle, rien de tel qu'un emploi correct des mots d'un langage. Un langage, comme le dit Kripke, semble « impossible, voire inintelligible ${ }^{4}$. » Kripke soutient d'autre part qu'il ne peut y avoir pour Wittgenstein qu'une solution «sceptique » (par opposition à une solution « directe ») du paradoxe. Une solution directe tente de montrer la fausseté de la conclusion paradoxale alors qu'une solution sceptique vise seulement à établir la « légitimité », pour employer le terme de Kripke, et non la vérité (au sens réaliste du concept de vérité) d'assertions telles que « Jean signifie par "plus" la fonction addition » (ou bien « Écrire "I002, I004, I006..." est la seule façon correcte de poursuivre la série “... 996, 998, ı000...” »).

3. Les références aux ouvrages de Wittgenstein sont données dans le texte entre parenthèses. Les abréviations utilisées seront les suivantes : «PU » pour Philosophische Untersuchungen, éd. par G.E.M. Anscombe et R. Rhees, Francfort. Suhrkamp, Ig69; «WLC » pour Wittgenstein's Lectures, Cambridge 1932-r935, ed. par A. Ambrose, Chicago. The University of Chicago Press. 1982; «WLFM » pour Wittgenstein's Lectures on the Foundations of Mathematics, ed. par C. Diamond, Chicago, The University of Chicago Press, 1989; «PG » pour Philosophische Grammatik, éd. par R. Rhees. Francfort, Suhrkamp, 1969; et « $B B »$ pour The Blue and Broun Books, Oxford, Basil Blackwell, $2^{\mathrm{P}}$ éd., 1969. Les références aux Recherches philosophiques ( $\mathrm{I}^{\text {tere }}$ partie) donnent le numéro de paragraphe.

4. WRPL, p. 62. 
Je présenterai d'abord dans ce qui suit une interprétation des remarques des Recherches sur les règles ( $P U$ i85-242) dans lesquelles Wittgenstein fait une critique d'une tentative de solution directe du paradoxe (section I). Jexpliquerai ensuite en quoi consiste sa solution « sceptique » (section 2).

\section{1. «la signification estl'usage»}

Pour décrire le rôle du paradoxe dans les remarques des paragraphes 185 à 242 , je rappellerai en premier lieu les grandes lignes de la conception du langage des Recherches.

Dans le Tractatus, Wittgenstein soutenait que tous les mots d'un langage, à l'exception des constantes logiques, ont pour fonction de « nommer » quelque chose. Comme il le dit dans les Recherches, les mots, d'après cette conception du langage, « nomment des objets [...]. Chacun [...] a une signification. Cette signification est coordonnée au mot. Elle est l'objet auquel le mot se rapporte » $\left(P U_{\mathrm{I}}\right)$. Pour montrer que cette façon de voir est erronée, il décrit le jeu de langage très simple suivant. Quelqu'un (A) demande à quelqu'un d'autre (B) de se rendre à une épicerie. A remet à B un bout de papier sur lequel on lit les mots " cinq pommes rouges ». B l'apporte avec lui et, arrivé à l'épicerie, il le montre à l'employé. Celui-ci ouvre alors un tiroir portant une étiquette sur laquelle on lit le mot « pommes »; il cherche ensuite le mot « rouge » sur une table d'échantillons de couleurs et, pour chacun des nombres de un à cinq qu'il énumère, il retire une pomme de la couleur correspondant à celle de l'échantillon. Il les remet ensuite à B. « C'est ainsi et d'autres façons semblables, dit Wittgenstein, que l'on opère avec des mots $\gg(P U$ I).

L'exemple est censé montrer en premier lieu que tous les mots n'ont pas pour rôle de nommer des « objets ». Le mot « pomme » est employé comme un nom, mais ce n'est pas le cas de « rouge » (qui est utilisé non pas pour nommer mais pour « caractériser » des choses) ni de « cinq » (qui n'est pas seulement employé ici pour nommer des objets ou groupes d'objets mais, veut dire apparemment Wittgenstein, pour les compterl. Chaque mot dans le jeu a une fonction, un certain type d'usage caractéristique, une « grammaire » (cf. $P G$ p. 6I et $P U$ Io). La signification d'un mot, d'autre part, n'est pas l'objet auquel il est « coordonné » mais son « usage ». Par l'« usage », Wittgenstein veut dire non seulement la fonction caractéristique d'un certain type de mots (des « noms de nombres », des « noms de couleurs », etc.), mais le rôle d'ensemble du mot dans un jeu de langage (qui comprend à la fois des « actes de langage » et des actions non linguistiques). Si, pour expliquer la signification de « cinq pommes rouges », on donne une description 
complète du jeu, y compris des circonstances dans lesquelles les mots sont prononcés (lus, écrits, etc.), des actions des participants, des instruments utilisés, des effets des actions (produits sur les participants ou sur les objets) etc., il n'y a pas lieu de se demander, en plus de ce qui a déjà été dit, en quoi consiste (quelle est) la signification des trois mots en question. Comprendre la grammaire de l'expression «l'explication de la signification (d'un mot) », c'est-à-dire comprendre la grammaire de l'expression « l'explication de l'usage (d'un mot) » (PG p. 59-60), peut «Inousl guérir de la tentation de chercher un objet que Inous appellerions] "la signification" du mot » (BB p.I).

On peut difficilement exagérer l'importance dans les Recherches du slogan d'après lequel la signification d'un mot (ou d'un énoncé) est son « usage » dans des jeux de langage ( $f f . P U$ 43). Wittgenstein l'oppose à deux idées fausses (selon lui) au sujet du concept de signification: celle qui veut que la signification d'un mot soit l'objet qu'il désigne (cf. PU I) et celle voulant que la signification soit une sorte d'entité psychique qu'on lui associe (une pensée, une image mentale, etc.) ou, en général, l'idée d'après laquelle « signifier » ou « comprendre » (un mot, une proposition, un signe) sont des activités de l'« esprit » chez les utilisateurs du langage, les accompagnements cachés des opérations visibles effectuées à l'aide des signes ( $c f . B B$ p. I7-18). Dans les paragraphes 138 à I84, qui traitent du concept de compréhension, il mentionne plusieurs expressions d'une façon de voir comme celle-là, en particulier les deux suivantes :

I. Parfois, on dit que, pour que quelqu'un comprenne un mot - par exemple «cube »- prononcé par une personne, il doit lui venir à l'esprit l'image mentale d'un cube; si par exemple il a reçu l'ordre d'aller chercher un objet de forme cubique, l'image lui venant à l'esprit est supposée déterminer la façon dont il va agir, elle fixe l'usage du mot, ici le comportement consistant à obéir à l'ordre (cf. PU I39-142). La difficulté dans cette façon de voir est que l'image peut toujours en fait être «projetée » de plusieurs façons différentes. On peut penser à une «méthode de projection » telle que celle-ci pourrait s'accorder non avec un objet de forme cubique mais avec un objet ayant la forme d'un prisme triangulaire : l'image « nous suggerlel une certaine application », celle qui s'accorde avec la méthode de projection « courante » ou « naturelle », c'est-à-dire avec l'application qu'en feraient la plupart des gens, « mais Inous pouvonsl aussi l'appliquer d'une autre façon » (PU i3g). Il est possible en ce sens que la même image vienne à l'esprit de deux locuteurs mais qu'ils emploient le mot (obéissent à l'ordre) de deux façons différentes. Est-ce que la signification du mot ou de l'ordre est la même pour les deux ? On dirait au contraire qu'elle est différente parce 
qu'ils ne font pas le même «usage » du mot. L'important, ce n'est pas ce qui se passe dans l'esprit des locuteurs quand ils entendent prononcer un mot, mais l'« usage » qu'ils en font (cf. PU I40).

II Le verbe « comprendre » est employé souvent non dans un sens «épisodique » pour désigner une occurrence mentale telle qu'une image, mais dans un sens « dispositionnel ». On dit en cet autre sens que quelqu'un comprend un mot (connait sa signification) seulement s'il possède une certaine capacité, la capacité de l'employer. Mais on tend à assimiler cette capacité (ou disposition) à un état (inconscient) de l'« esprit » ou de l'« appareil psychique », lequel est censé « expliqueirl les manifestations de lcette compréhensionl » (PU I49) : «lulne disposition est conçue comme quelque chose qui est toujours là [et] dont découlent les comportements. Elle est analogue à la structure d'une machine et à son comportement » (WLC p. gi). Wittgenstein rejette cette façon de concevoir les capacités. Les critères qu'on utilise pour établir que quelqu'un possède une capacité sont liès à des comportements (ici à l'usage du langage) et non à l'existence d'un état caché de son esprit ou de son cerveau. Si on peut dire que quelqu'un possède une capacité sans rien savoir (et sans qu'il soit nécessaire de faire des hypothèses) sur ce qui se passe dans son esprit, le concept d'une capacité (ici celui de compréhension) est indépendant de celui d'une condition de l'esprit ou d'un mécanisme psychique et il est lié (conceptuellement) à celui d'usage (cf. PU I49; PU I57-58 et WLC p. 92-93).

Après ces remarques, Wittgenstein reprend la question des règles $\left(P U\right.$ I $\left.85^{-242}\right)$. Les concepts de signification et de compréhension renvoient au concept d'usage. Or, utiliser un signe « en accord avec sa signification », c'est l'employer comme on doit le faire, c'est-à-dire en faire un usage correct ou conforme à des régles (la distinction usage correct/usage incorrect est indissociable du concept d'une régle). Donc, se demander «Qu'est-ce que faire un usage correct d'un signe ?» mène à poser l'autre question : «Qu'est-ce qu'une application correcte d'une règle? ». On sait par ailleurs que le problème de la normativité des règles est le problème soulevé par le paradoxe («Comment appliquer une règle correctement si n'importe quelle action est compatible avec la règle? $\gg$. Wittgenstein veut d'abord montrer qu'il ne peut y avoir une solution directe du paradoxe.

L'idée suivant laquelle il y a toujours une seule manière « correcte » d'appliquer une regle s'exprime entre autres par l'affirmation qu'une fois fixé le contenu de la règle, ses applications correctes se trouvent aussi fixées : toutes les « conséquences » futures et possibles de la règle « préexistent » à son application avant même qu'elles aient été tirées (cf. $P U$ I88, 218 et 219). La même idée s'exprime parfois comme suit : lorsque 
quelqu'un obéit à une règle, il ne fait que « développer » ce qu'elle « contient » déjà - un peu comme s'il tirait d'un tiroir un collier de perles (cf. $P G$ p. 55) - de sorte qu'il n'a aucun « choix », il agit en quelque sorte « contraint » par la règle (cf. $P U$ 23I). Or, un corollaire de la conclusion sceptique est qu'une règle ne "contient » pas d'avance toutes ses applications; écrire « I000, I002, 1004, ... » n'est pas la seule façon possible de suivre la règle « Pour tout $n$, écrire $n+2$ ». Quelqu'un pourrait « décider » (cf. $P U$ I86) d'écrire « IOO4 » au lieu de «IOO2 » après avoir écrit « IOOO » dans la mesure où il pourrait « choisir » d'interpréter la règle en ayant recours à une autre règle (d'un autre niveau) et celle-ci au moyen d'une troisième règle, etc. Mais on pourrait objecter que, si la règle telle que formulée (par le maître) peut toujours s'interpréter d'une multitude de façons différentes, dans la mesure où les formulations d'une règle laissent toujours place à toutes sortes d'interprétations, ce n'est pas le cas de la règle telle que le maître la comprend. Lorsqu'il donne l'ordre « Ajoutez 2 successivement à chacun des nombres! », le maître « veut dire » que l'élève doit écrire entre autres « 1002 » après Iooo et non pas « IOO4»; c'est ce qu'il « signifie » quand il lui demande de poursuivre la série. L'important, c'est la façon dont le maître « comprend » (meint) la rẻgle, ce qu'il « veut dire » quand il l'énonce ( $c f . P U \mathrm{I} 87$ ). Au sujet de cette façon de voir, à laquelle j'ai déjà fait allusion ${ }^{5}$, Wittgenstein écrit :

I... lVlotre idée était que cet acte de signifier l'ordre ljenes Meinen des Befehls] avait d'une certaine façon déjà franchi toutes ces étapes [dans le développement de la suite de nombresl : qu'en signifiant ll'ordrel votre esprit s'était pour ainsi dire envolé au devant et les avait toutes traversées avant que vous soyez parvenu physiquement à celle-ci ou à celle-là.

Ainsi, vous étiez enclin à employer des expressions telles que : «Les étapes sont en réalité déjà franchies, avant même que je les aie

5. Il semble qu'il y ait pour Wittgenstein une similitude entre l'opinion d'après laquelle le contenu des règles telles que « signifiées » par leurs utilisateurs - par opposition aux règles-telles-que-formulées-verbalement - est déterminé dans leur esprit, et le point de vue du Tractatus selon lequel il y a des règles «strictes et claires » de la structure logique des propositions, des règles qui, dit-il, sont « cachées » dans le « médium de la compréhension » ou de la « signification » (cf. $P U$ Io2). Le paragraphe 8I (dans la première série de remarques sur les règles) fait probablement allusion a ce point de vue. Wittgenstein écrit : quand « nous aurons obtenu plus de clarté sur les concepts de compréhension, de signification et de pensée », nous verrons mieux alors « ce qui peut nous conduire (et m'a conduit) à penser que quelqu'un qui énonce une proposition et la signifie ou la comprend opère ce faisant un calcul d'après des règles dèterminees [nach bestimmten Regeln]. » Il semble que Wittgenstein pense aussi bien à ce qu'il va dire un peu plus loin ( $c f$. en particulier $P U$ IO2 qu'à la seconde série de remarques sur les règles. 
traversées par écrit, oralement ou en pensée. » Et cela [vous] était apparu comme si elles étaient prédéterminées de façon absolument unique, anticipées - comme seul l'acte de signifier [das Meinen] peut anticiper la réalité ( $P U$ I 88 ).

Dans l'acte de « signifier », ce que quelqu'un doit faire pour suivre correctement la règle est entièrement déterminé. Si l'élève pouvait appréhender le «contenu pensé » de la règle (la règle telle que la « signifie » le maître), il ne pourrait pas la suivre autrement que de la façon dont le maître veut qu'il la suive : il n'aurait plus le choix, il ne pourrait pas écrire « IOO4 » après avoir écrit « IoOO » (cf. PU z Io et WLC p. r32). Dans un de ses cours, Wittgenstein dit:

L'affirmation d'après laquelle le maitre a « signifié » quelque chose au moment où il enseignait la règle suggère qu'un autre processus se déroulait pendant qu'il l'enseignait ou bien que, même s'il n'y avait pas de processus correspondant à chacune de ces étapes, il y avait un processus renfermant toutes les étapes et dont elles découlent toutes, un processus étrange contenant toutes ces étapes non encore nées [all these unborn stepsl. Si len effet] ce que le maître signifiait ne llesl contenait pas toutes, comment expliquer qu'il ait su immédiatement que l'élève a commis une erreur? ( WLC p. I3I)

La réponse à cette tentative de solution directe du paradoxe est contenue pour une part dans les remarques sur le concept de compréhension (ce qui explique au moins en partie que le problème de la normativité des règles soit traité seulement à la suite de ces remarques). La question est donc de savoir si le contenu « pensé » ou « signifié », par opposition au contenu exprimé de la règle, est ce qui fixe son application correcte; ou bien il est de savoir si la signification d'un mot, entendue au sens d'une « idée », d'une image mentale ou d'un état de l'esprit des locuteurs, est ce qui fixe son usage correct (ce que dit Wittgenstein au sujet des concepts de «signification », d'image mentale, d'«idée », etc., vaut aussi, mutatis mutandis, pour celui de « contenu pensé » de la règlel. L'essentiel de sa critique se trouve dans le paragraphe i93, où il s'attaque à l'idée d'après laquelle la « signification » est une condition, un état dispositionnel de l'esprit ou un mécanisme « contenant » en lui-même toutes ses possibilitès de mouvement au sens où on dit de la « signification » d'un mot qu'elle « contient » tous ses usages corrects :

La machine comme symbole de son action : la machine l...l semble déjà renfermer en elle-même son mode d'action [Wirkungsweise]. Qu'est-ce à dire ? - Si nous connaissons la machine, tout le reste, c'est-à-dire le mouvement qu'elle va effectuer, semble déjà complètement déterminé.

Nous parlons comme si lsesl parties ne pouvaient se mouvoir que de cette façon [...]. Comment - oublions-nous la possibilité qu'elles puissent plier, se briser, fondre, etc. ? Oui, dans bien des cas nous ne 
pensons pas du tout à cela. Nous utilisons une machine, ou bien le dessin [Bild] d'une machine, comme symbole d'un certain type d'action. Nous communiquons par exemple à quelqu'un ce dessin et supposons qu'il va en dériver le mouvement des parties. [...]

« La machine semble déjà renfermer en elle-même son mode d'action » veut dire : nous sommes enclins à comparer les mouvements futurs de la machine l...l à des objets déjà déposés dans un tiroir et que nous retirons ensuite. - Mais nous ne parlons pas comme cela quand il s'agit de prédire le comportement réel d'une machine. Car nous n'oublions pas alors en général la possibilité de la déformation des parties, etc. Nous parlons de cette façon, par contre, quand nous nous étonnons de ce qu'il est possible d'utiliser la machine comme symbole de son action, - car elle peut très bien aussi se mouvoir d'une toute autre façon. [...]

Quand nous nous rappelons que la machine aurait pu également se mouvoir d'une autre façon, il peut nous sembler que la machine en tant que symbole contienne en elle-même son mode d'action d'une façon bien plus détenminée que la machine réelle. I...I

Dans un cours, de façon plus explicite, Wittgenstein dit:

Une notion nous a laissé perplexe llors d'un cours précédentl, la notion d'après laquelle, quand nous comprenons un mot, notre idée nous fait employer [makes us use] le mot d'une certaine façon particulière. C'est comme si l'idée contenait l'usage l...l. J'ai voulu faire remonter cette notion à celle d'un mécanisme. Or ce que fait un mécanisme ne découle pas Idoes not followl de ce qu'il est en aucun sens important. Ce que va faire le mécanisme ne peut qu'être conjecturé à partir de ce qu'il est l.... Avant de réaliser que nous pouvons seulement inférer de façon hypothétique ce que fait un mécanisme de ce qu'il est, nous tendons à comparer une idée à un mécanisme. Cette notion doit être écartée ( $W L C$ p. 87 ).

La « signification » (le contenu de la règle tel que «signifié » par le maître) est une sorte d'état dispositionnel (un mécanisme) de l'esprit « contenant » l'usage correct des mots (les applications correctes de la règle) au sens où on dit d'une machine qu'elle « contient » virtuellement les mouvements de ses parties (ou les « possibilités » de son mouvement ( $c$. PU I94)). Le problème dans cette façon de voir ne réside pas tellement dans le fait qu'on peut seulement faire des suppositions sur le mécanisme en question (bien que ce soit le cas) que dans le fait que, même si on suppose connue sa nature (sa structure, son organisation), on ne peut jamais être certain que ses parties vont se mouvoir de la façon dont on croit qu'elles vont le faire, pour la raison mentionnée dans le paragraphe ig3 (et à laquelle le texte du cours fait allusion) - on peut seulement « inférer de façon hypothétique », comme dit Wittgenstein, ce que va faire le mécanisme. Assimiler la « signification » à un état de l'appareil psychique, par conséquent, n'explique pas ce qu'on cherche à expliquer : le fait (prétendu) qu'il y a une seule façon 
« correcte » d'utiliser un mot, une seule façon correcte de poursuivre la série.

Mais, d'un autre côté, on pourrait considérer la machine comme symbole de son mouvement et représentée par un « dessin » (Bild). Il semble qu'à partir du dessin de la machine on ne puisse « dériver » qu'une série de mouvements du mécanisme - quelqu'un à qui on montrerait le dessin serait capable de « dériver » tous ses mouvements. Si on peut seulement faire des conjectures sur le mouvement de la machine réelle (dont les parties sont susceptibles de se briser, de fondre, etc.), on peut apparemment prédire de façon certaine le mouvement des parties de la machine considérée comme symbole (comme on le lit dans le paragraphe 193. « [...] il peut nous sembler que la machine en tant que symbole contienne en elle-même son mode d'action d'une façon bien plus déterminée que la machine réelle »). Et c'est ce qui expliquerait qu' il n'y ait qu'une façon correcte d'appliquer une règle. Dans le même cours, Wittgenstein répond à cela comme suit (je ne cite qu'un fragment d'un développement beaucoup plus long):

Ces propositions qui semblent analogues à des propositions empiriques au sujet de mécanismes, par exemple lla propositionl que telle ou telle action ou usage [d'un motl va [...] « découler » (follow froml d'une idée, ne sont pas réellement analogues à des propositions empiriques, car ce sont des regles. Et c'est pourquoi elles ont l'apparence de la certitude que n'ont pas les propositions Ihypothétiquesl au sujet de mécanismes I... (WLC p. 87).

Si la proposition «L'action $X$ "découle" de l'idée $Y$ » est une proposition au sujet d'un mécanisme, alors ce n'est qu'une conjecture, une proposition empirique, et elle n'est certaine qu'en apparence : on ne peut être certain que les parties de la machine vont effectivement se mouvoir de la façon dont on a prédit qu'elles vont le faire. Si par contre elle est l'expression d'une régle, comme c'est le cas du dessin du paragraphe r93, donc si elle est considérée comme « symbole », alors on n'a rien expliqué : il s'agissait, en faisant appel à l'idée d'un mécanisme, de comprendre pourquoi une régle ne peut avoir qu'une application « correcte ». En somme, ou bien la règle-telle-que-la-pense-le-maitre est un mécanisme (une condition, une structure) de l'appareil psychique et, dans ce cas, on ne voit pas pourquoi elle ne devrait avoir qu'une application « correcte » (pour la raison mentionnée dans le paragraphe i93); ou bien, si on la considère comme « symbole », elle est semblable aux autres règles (à ceci près qu'elle est supposée se trouver dans l'« esprit » du maître) et on n'a toujours pas réussi à expliquer ce qu'on cherchait à expliquer (cf. aussi WLFM p. 183 $)^{6}$.

6. Kripke discute un certain nombre de suggestions de solution directe du paradoxe 
En résumé, une des idées fondamentales des Recherches est qu'on a tout dit sur la signification d'un mot si on a décrit l'«usage »du mot. c'est-à-dire les actions des locuteurs (« la façon dont ils opèrent avec les signes $\gg[P U \mathrm{I}])$, les effets de leurs actions, etc. quand ils l'emploient dans leurs jeux de langage ${ }^{7}$. La signification n'est pas de l'ordre des choses qui sont « cachées » dans l'esprit des locuteurs : tout dans le phénomène du langage est « public ». D’autre part, la compréhension d'un mot est une capacité d'agir, la capacité d'en faire un usage correct

dont certaines sont similaires à celles que mentionne Wittgenstein (cf. WRPL p. 22-54). Il cite entre autres une solution qui fait appel à l'idée de disposition (cf. WRPL P. 22-37) et il remarque, à mon avis avec raison, que Wittgenstein rejette cette solution (cf. WRPL p. 35, note 24). Des commentateurs des Recherches l'ont critiqué sur ce point. C. McGinn, par exemple, écrit : «Wittgenstein fait des remarques qui viennent en fait à lappui de la suggestion dispositionnelle contre laquelle s'élève Kripke l...J. » (Wittgenstein on Meaning, Oxford, Basil Blackwell, 1984. p. 74). McGinn cite en particulier le paragraphe 187 des Recherches dont il ressort selon lui que Wittgenstein souscrit à une analyse dispositionnelle du concept de signification (sur la base d'énoncés du type « Jean signifie par "plus" la fonction addition parce que si on lui demandait quelle est la somme de 57 et de 68 il répondrait "125" »). Je pense que Wittgenstein soutient en fait dans ce passage exactement le contraire : ce n'est, dit-il, qu'une « hypothèse » que Jean répondrait « I25 ». Cf. BB P. I4I-I42, un texte dont le contenu est proche de celui de $P U$ I87 mais qui est beaucoup plus explicite. Wittgenstein écrit $(B B \mathrm{p} .142)$ : « Mais que veut dire suivre la rẻgle correctement ? Quand et comment décider quelle est l'étape correcte en un certain point [de la sériel ? - 'L'étape correctel...] est ce qui s'accorde avec la règle telle qu'elle était signifiée Imeant, intendedl." - Je suppose que lvotrel idée est la suivante : Lorsque vous avez donné la règle "Ajoutez I" et l'avez signifiée, vous vouliez qu'il écrive Ior après I0O, 199 après 198, IO4I après Io40, et ainsi de suite. Mais comment avezvous effectué tous ces actes de signification (je suppose en nombre infini) quand vous lui avez donné la règle? [Ou bien] diriez-vous qu'il y avait alors seulement un acte de signification, mais dont tous les autres ont découlé [...] ? Vous pourriez dire: "Je savais sûrement quand je lui ai donné la règle qu'il devait lthat I meant him tol écrire Ior après 100". Mais ici vous êtes induit en erreur par la grammaire du verbe "savoir". Est-ce que savoir cela était un acte mental par lequel vous avez fait à ce moment-là le passage, par exemple, de roo à ıo [... ? Dans ce cas, demandez-vous combien de ces actes vous avez effectué quand vous lui avez donné la règle. Ou bien voulez-vous dire, par ce savoir, une sorte de disposition? - Dans ce cas, seule l'expérience peut nous apprendre de quelle disposition il s'agissait lwhat it was a disposition forl. » On lit un peu plus loin : «l...l le temps passé du verbe "signifier" suggère qu'un acte particulier de signification a été effectué lorsqu'a été donnée la règle. bien qu'en fait l'expression employée lc'est-à-dire "I meant you to write Ioı"] ne fasse pas allusion à un tel acte. Le passé [du verbe "signifier"] pourrait être expliqué en donnant à la proposition la forme “Si vous m'aviez demandé auparavant ce que je voulais faire en ce point, j'aurais répondu..." Mais c'est une hypothèse que vous auriez ldonné cette réponsel. »

7. Wittgenstein ècrit : «[n]os paroles l'est-à-dire nos activités linguistiques] 
(elle ne consiste pas dans la présence d'une image mentale, d'un état dispositionnel de l'appareil psychique ou d'autre chose de semblable). Mais qu'est-ce qu'un usage correct ? Il découle du paradoxe des règles qu'il n'y a rien de tel qu'une distinction entre des applications correctes et des applications incorrectes d'une règle. Il faut donc trouver une «solution ». Wittgenstein rejette la solution suivant laquelle une application correcte est fixée par ce qui se trouve dans l'« esprit » des utilisateurs. La règle pourrait se trouver quelque part « dans leur esprit » (cachée dans le « médium de la signification ») ou formulée verbalement ou bien à l'aide d'un dessin (donc considérée comme «symbole »), on pourra toujours l'interprèter de multiples façons différentes (cf. aussi $P U$ I4I). Ce qui est vrai d'une disposition (d'un état non conscient de l'appareil psychiquel est vrai aussi de tous les phénomènes mentaux conscients : une image mentale ne « contient » pas non plus « en elle-même » son application; il est possible de l'« appliquer » en suivant des « méthodes de projection » différentes et incompatibles ${ }^{8}$.

\section{2.la solution sceptique}

Je pense qu'il est plausible de supposer qu'il n'y a pas pour Wittgenstein d'autre solution directe du paradoxe. Une solution sceptique, d'autre part, n'a pas pour but de réfuter la conclusion paradoxale mais de montrer en quoi sont « légitimes », comme dit Kripke , des assertions (courantes) telles que « Jean "signifie" par "plus" la

reçoivent leur sens de nos autres actions », Ueber Gewissheit. Francfort, Suhrkamp, I969, p. 229).

8. Kripke décrit comme suit le plan des Recherches du début jusqu'aux remarques sur le problème du langage privé (cf. WRPL, p. 78-79) : (a) PU I`r37 : Wittgenstein fait la critique de la conception du langage du Tractatus et il en suggère une autre; (b) PU $138^{-242}$ (« les paragraphes centraux des Recherches », dit Kripke IWRPL, p. 79l) : il introduit le paradoxe et sa solution sceptique; (c) PU 243 et suivants : il montre l'impossibilité d'un langage privé. La relation entre (a) et (b) serait celle-ci : Wittgenstein fait en (a) la critique de la sémantique vériconditionnelle (ou « référentielle ») du Tractatus et il suggère pour la remplacer une sémantique fondée sur le concept de « conditions de justification » ou de « conditions d'assertabilité ». Kripke cite M. Dummett qui écrit ; les Recherches " contiennent implicitement le rejet du point de vue classique (réalistel de Frege et Wittgenstein [dans le Tractatus] d'après lequel la forme générale d'explication de la signification [d'une proposition] consiste en un énoncé de Isesl conditions de vérité » k Wittgenstein's Philosophy of Mathematics », repris dans G. Pitcher (éd.), Wittgenstein : «The Philosophical Investigations », Doubleday, Garden City, N.Y., 1966, p. 446-447. Cf. aussi « Frege and Wittgenstein », dans I. Block (éd.), Perspectives on the Philosophy of Wittgenstein, The MIT Press, Cambridge, Mass., I984. P. 39-40. Il 
fonction addition » (ou « L'élève ne développe pas "correctement" la suite quand il écrit "I000, I004, I008 ..." »). On peut aussi supposer qu'une solution sceptique s'appuie seulement sur des faits relatifs à l'usage du langage par des locuteurs (par opposition à ce qui peut se passer dans leur esprit).

Je présenterai la solution telle que l'interprète Kripke en citant des textes des Recherches. Je signalerai ensuite une difficulté dans la conception « communautaire » du langage que présuppose son interprétation. Je suggérerai après cela une autre interprétation qui, je pense, évite la difficulté et semble compatible avec les textes cités.

Une solution sceptique doit rendre compte de deux genres de faits au sujet de l'idée de correction. Premièrement, on dit que des personnes ont raison lorsqu'elles disent appliquer correctement une règle (c'est le maître et non l'élève qui a raison quand il fait observer que « IOOO, I0O4, I008, ... » n'est pas la façon correcte de développer la sériel. Deuxièmement, on considère que quelqu'un suit correctement (ou incorrectement) une règle peu importe ce qu'il croit à ce sujet : il pourrait croire l'appliquer correctement alors que ce n'est pas le cas ou bien croire avoir commis une erreur en l'appliquant alors qu'il n'a en fait commis aucune erreur. Comme le dit Wittgenstein, croire suivre une règle (correctement) n'est pas la même chose que la suivre (correctement) (cf. $P U$ 202); on

semble que la «theorie de la signification » des Recherches (une théorie de la « signification-usage ») soit selon Dummett une sémantique non vériconditionnelle (ou « non réaliste ») et, soutient Kripke, c'est précisément une sémantique non vériconditionnelle que suppose la solution sceptique : l'énoncè « Jean signifie la fonction addition par "plus" » n'est ni vrai ni faux (il n'y a pas de « fait déterminé » qui le rend vrai ou faux - il est dénué de conditions de véritél mais il a des «conditions d'assertabilité » (telles qu'il peut être asserté ou nié de façon justifiée). Le fait que l'énoncé possède des conditions d'assertabilité montre par conséquent sa « légitimité » (cf. WRPL p. 73). Cela expliquerait que l'exposé du paradoxe et de sa solution soit précédé dans les Recherches d'une critique de la conception du langage du Tractatus. Je ferai là-dessus seulement deux remarques. PTemièrement, il n'est pas évident (pour le moins) que la théorie de la signification des Recherches soit une sémantique « non réaliste » au sens de Dummett. Deuxièmement, si Wittgenstein rejetait complètement la sémantique « réaliste » du Tractatus (comme le suppose apparemment Kripke), la conséquence en serait qu'aucun énoncé déclaratif n'a de conditions de vérité - ce qui n'est pas très plausible : le sceptique sémantique a peut-être raison de prétendre que « Jean signifie par "plus" la fonction addition » n'est ni vrai ni faux, mais il est douteux qu'il dise la même chose d'un énoncé tel que (disons) « Ia table qui se trouve devant moi est blanche ».

9. Cf. WRPL, p. 77 . 
pourrait en ce sens parler d'une application « objectivement » correcte d'une règle.

La solution, dans l'interprétation de Kripke, est la suivante ${ }^{\text {IO }}$. Supposons, que quelqu'un comprenne la règle $R_{I}$ en l'interprétant à l'aide de $R 2$. A la question «Quelle est la somme de i et de 2 ? », par exemple, il va répondre « 3 » et si on lui demande combien font 57 et 68 , sa réponse sera « 5 ». Considéré comme locuteur isolé (ou unique), rien ne nous permet de dire qu'il emploie «plus » (ou « somme de ») correctement ou non. Il peut être certain qu'en répondant « $5 »$ il en a fait un emploi correct. Mais la règle pourrait être interprétée au moyen d'une autre règle, par exemple $R_{3}$ (telle que la réponse « correcte » serait, disons, 6 et non 5). La situation n'est pas différente s'il existe un deuxième utilisateur de «plus ». Celui-ci pourrait comprendre RI en l'interprétant à l'aide d'une règle différente de $R 2$, mais on n'a aucune raison de prétendre que c'est lui qui emploie « plus » correctement. D'un autre côté, supposons qu'il existe plusieurs utilisateurs de « plus » et que, sauf exceptions, ils font tous un même usage de «plus » : ils l'emploient en accord avec $R_{1}$ et sans en donner une interprétation de niveau 2. (Toute règle peut être interprétée, expliquée ou comprise au moyen d'une autre règle mais, dit Wittgenstein, les gens suivent le plus souvent leurs règles sans penser à les interpréter ou à les expliquer : ils agis sent simplement en accord avec les règles ${ }^{\mathrm{II}}$.) S'ils font tous la même application de $R_{I}$ (agissent de la même façon quand ils la suivent), c'est ce qu'on va appeler (et ce qu'eux-mêmes appellent) une application « correcte » de RI (pour ce groupe d'individus). « Correct», quand on parle de l'application d'une règle, ne veut rien dire d'autre que « tel que l'emploient la majorité de ses utilisateurs ».

En quel sens l'élève a-t-il commis une erreur en écrivant « IoO4, I008, ... » après « Io0o »? En quel sens est-ce une erreur de répondre « 5 » à la question « Combien font 57 et 68 ? »? En ce sens que la majorité des utilisateurs compétents de «plus » l'emploient en suivant RI et qu'une application « correcte » de RI, si elle n'est pas interprétée en ayant recours à une autre règle, consiste justement à répondre « I25». Il n'y a pas d'usage intrinsèquement correct de « plus », seulement des usages qu'on peut qualifier de « corrects » relativement à des groupes d'individus (ce qui veut dire que la façon, pour nous incorrecte, dont l'élève poursuit la série pourrait être correcte pour d'autres locuteurs). Par ailleurs, la distinction correct/incorrect est objective en ce sens que,

I0. Jadapte l'exposé de Kripke à mon interprétation du paradoxe.

II. Cf. première partie, section 2. 
si un des utilisateurs de «plus » commet sans le savoir une erreur en l'employant, son usage était incorrect dans la mesure où d'autres utilisateurs compétents n'auraient pas fait cet usage-là (ou dans la mesure où un autre locuteur aurait pu corriger son erreur). Un usage n'est donc correct (« objectivement ») que relativement à des groupes de locuteurs.

Peut-on citer des textes à l'appui de cette interprétation ? Les passages portant sur la solution du paradoxe (dans la seconde série de remarques sur les règles) ne sont (malheureusement) pas très explicites mais on peut citer les paragraphes i8g et Igo, auxquelsj'ajouterai le paragraphe ig8. Je citerai (en partie seulement) ces textes et j'y renverrai ensuite par les chiffres entre crochets :

[I] [...] Nous employons l'expression : « les étapes sont déterminées [bestimmt] par la formule... [c'est-ä-dire la règle...l ». Comment [l'expressionl est-elle employée? - Nous pouvons par exemple nous référer au fait que des gens, à la suite d'un enseignement (d'un dressage (Abrichtungl), sont amenés à employer la formule $y=x 2$ lexprimant la règlel de telle façon que tous obtiennent toujours comme résultat la même valeur de $y$ s'ils ècrivent le même nombre pour $\mathbf{x}$. Ou bien nous pouvons dire : « Ces gens sont ainsi dressés labgerichtetl que, parvenus en un même point, ils vont tous écrire ensuite le même nombre s'ils ont reçu l'ordre " +3 " 》. Nous pourrions [aussi] exprimer cela comme suit : l'ordre $«+3$ » détermine complètement pour ces personnes le passage de tout nombre au suivant. (Par opposition à d'autres qui ne savent pas ce qu'elles ont à faire quand elles reçoivent cet ordre; ou bien qui réagissent à l'ordre avec une certitude complète mais chacune d'une manière différente.) (...) (PU I8g).

[2] On peut maintenant dire : « La façon dont est comprise lgemeintl la formule est ce qui détermine lquel nombre on doit écrire après un autrel. » Quel est le critère de la façon dont est comprise la formule ? C'est par exemple la façon dont nous l'employons constamment Istaendig gebrauchenl, la façon dont on nous a enseignè à l'employer [...] (PU 190).

[3l l... Posons-nous la question : qu'est-ce que l'expression d'une règle - disons un panneau indicateur - a à voir avec mes actions ? Quel genre de rapport y a-t-il ici ? - Eh bien, ce pourrait être celui-ci : j'ai été dressé [abgerichtet] à réagir d'une certaine façon à ce signe et c'est de cette façon que maintenant je réagis.

Mais vous avez seulement donné en disant cela une relation de cause à effet, vous avez indiqué seulement comment nous en sommes venus, comme maintenant, à nous guider d'après le panneau indicateur; vous n'avez pas dit en quoi consiste leigentlich bestehtl) ce « suivre-le-signe ». Non, j'ai suggéré aussi que quelqu'un ne se guide d'après le panneau que dans la mesure où il existe un usage constant leinen staendigen Gebrauchl, une coutume (PU I I8). 
[3] fait probablement allusion à [I] et à [2]. Comme on le lit en [3], [I] donne une explication (ou un schéma d'explication) causale du fait que ceux qui suivent un règle l'appliquent tous de la même façon let c'est sans doute aussi une explication de ce qu'ils ont l'impression que la règle les « contraint » à agir d'une certaine manière). Ensuite, l'alinéa [2] dit en quoi consiste « suivre-une-règle », ćest-à-dire en quoi consiste la suivre correctement (dire en quoi « consiste » suivre une règle n'est pas la même chose qu'expliquer causalement le fait que les gens en font tous une même application).

L'explication est la suivante : lorsque des individus sont entraînés («dressés 》) à obéir à une règle, tous (ou la très grande majorité d’entre eux) vont ensuite l'utiliser de la même façon. Ils vont également considérer cette manière d'utiliser la règle comme la façon « naturelle » de l'appliquer (ou peut-être comme la seule possible). S'ils avaient reçu un autre entraînement, ils en feraient une application différente et c'est celle-là qu'ils considéreraient comme l'application « correcte ». En bref, nous sommes ainsi constitués que, lorsque nous sommes entraînés à obéir à une règle (par exemple pour développer des suites de nombres), nous avons tous tendance à réagir de la mème façon (quand quelqu'un nous donne par exemple l'ordre « Ajoutez $3 ! \gg$ ).

Wittgenstein fait allusion en [2] à l'idée que la « signification » (au sens d'un quelque chose qui se passe dans l'esprit des locuteurs) est ce qui « détermine » l'application correcte d'une règle. Mais on doit plutôt selon lui poser la question suivante : quel est le critere de la façon dont est comprise (ou « signifiée ») une règle ? C'est-à-dire : quel est le critère de l'application correcte d'une règle (puisqu'employer un mot « en accord avec sa signification $\gg$, c'est l'employer correctement), ou encore : en quoi consiste une application correcte (qu'est-ce qui est « constitutif », comme il le dit en [3], d'une telle application) ? Au sens où il emploie le mot «critère », ce qui se passe dans l'esprit des locuteurs (par exemple une occurrence « priveee » telle quavoir une image présente à l'esprit) ne peut pas jouer le rôle d'un critère (constitutifl de l'emploi correct d'un mot (un critère est en effet interpersonnel ou au moins « public »). Il répond : est constitutive de l'application correcte d'une règle la façon dont ses utilisateurs l'appliquent toujours; une « application correcte » est l'application qu'en font constamment (staendig) ceux qui l'utilisent; autrement dit, c'est une « coutume » (cf. aussi PU 692).

On trouve dans les Recherches d'autres textes à l'appui de cette interprétation mais je citerai un passage de l'un des cours de Wittgenstein (qui sont souvent beaucoup plus clairs que ses textes écrits). Le passage est extrait d'un cours sur les fondements des mathématiques. 
On lit d'abord ceci : « [...] le point important est que nous faisons tous le MÊME usage [d'un mot]. Connaître lla] signification [de ce mot], c'est l'utiliser de la même façon que les autres l'utilisent. "De la façon correcte" ne veut rien dire. » (WLFM p. 183) Donc, « de la façon correcte » ne veut rien dire si ce n'est « de la façon dont tous l'utilisent». On lit ensuite ceci :

[I] Cela est lié à la question de savoir comment continuer la série des nombres cardinaux. Y a-t-il un critere de la continuation - de la manière correcte ou incorrecte Ide continuerl - sinon le fait que nous la continuons effectivement de cette façon-là, mis à part quelques excentriques qu'on peut négliger?

[2] Certes, nous donnons une règle générale pour continuer la série; mais cette règle générale pourrait être interprétée par une seconde règle, et cette seconde règle pourrait être interprétée par une troisième, et ainsi de suite. [...]

[3] Supposez que quelqu'un dise : « L'usage que je fais de la règle pour continuer la série dépend assurément de mon interprétation de la règle ou de la signification que je lui prête. » Mais le critère pour signifier quelque chose par la règle est-il la façon particulière d'employer la règle Ithe using of the rule in a certain wayl, ou bien est-ce une image ou une autre règle ou quelque chose du genre? Si c'est le ldernierl cas, alors on a toujours affaire à un symbole - lequel peut être réinterprèté strictement de n'importe quelle façon (WLFM p. 183; je souligne).

Il semble que dans les alinéas [I] et [3] Wittgenstein donne au mot « critère » le même sens que dans le paragraphe igo : le critère (ce qui est constitutif) de la façon correcte de développer une suite de nombres en conformité avec une règle est ce que font la majorité des utilisateurs compétents de la règle ( $c f$. aussi $P U$ 692).

[2] répond à une objection : celui qui défend une solution directe du paradoxe pourrait objecter que c'est la régle et non l'usage (l'application constante) qu'en font les gens qui montre en quoi consiste (et qui fixe) son application correcte. C'est la règle elle-même qui « détermine » comment on doit l'appliquer (qui « contient » la suite de nombres, etc.). En effet, la plupart de nos règles sont générales (« Pour tout nombre, écrire celui qui vient après le suivant $\gg$ ), et c'est pourquoi l'élève ne pouvait pas écrire « IOO4 » après avoir écrit « IOOO » (chacun des nombres de la série, veut dire l'objecteur, est « déterminé » par la règle). La réponse est contenue implicitement dans ce qui précède : une règle générale peut aussi être réinterprétée au moyen d'une autre règle.

[3] répond à une objection dont il a été question plus haut : est-ce que ce n'est pas la signification donnée à la règle, l'« interprétation » qu'on en donne (si on entend par « interprétation » un processus ayant lieu dans l'esprit de ses utilisateurs) qui détermine son usage correct? Wittgenstein répond: si la «signification » est quelque chose du genre 
d'une image ou d'une règle « dans l'esprit » de ses utilisateurs, alors, considérée comme symbole, l'image (ou la règle) peut être interprétée de bien des façons différentes ( $c$. ci-dessus, section I). Cela n'a aucune importance, comme il le répète souvent ( $c$. par exemple $P G$ p. gg), que l'image ou la règle soit formulée verbalement, qu'elle soit inscrite ou dessinée sur une feuille de papier ou qu'elle soit située quelque part « dans l'esprit » des locuteurs : le critère de la signification (du contenu) de la règle, ce qui est constitutif de son application correcte, c'est non pas quelque chose qui serait présent dans l'esprit de ses utilisateurs mais l'usage qu'ils en font.

Cette lecture de la solution sceptique, je pense, est plausible compte tenu de ces derniers textes, mais il semble qu'il y ait une difficulté dans la conception «communautaire »du langage que présup pose l'interprétation de Kripke. Pour montrer en quoi elle consiste, je rappellerai brièvement l'argument de Wittgenstein contre l'idée d'un « langage privé »- un argument qui, selon Kripke, est un corollaire de la solution sceptique.

Un langage privé se définit comme un langage qui ne peut (« logiquement ») être compris que par celui qui l'utilise. Wittgenstein invoque l'exemple d'un nom employé par un locuteur pour se référer à une sensation qu'il qualifie elle-même de « privée »: seul le locuteur peut savoir s'il a la sensation et personne d'autre n'est en mesure de savoir quelle est sa nature (sa qualité subjective ressentie). $S i \ll S »$ est le mot choisi pour s'y référer (un signe que le locuteur pourra utiliser par exemple pour noter sa récurrence dans son journal), alors « $S$ »est un nom privé.

Un langage (un mot) privé n'est pas possible parce qu'on ne peut faire dans un tel langage une distinction objective entre un usage correct et un usage incorrect d'un mot. Le locuteur du langage ne possède en effet aucun « critère de correction » pour contrôler son emploi de « $\mathrm{S}$ » (cf. $P U$ 258). Un « critère de correction » est un moyen indépendant (et interpersonnel, dit apparemment Kripkel pour dire si un mot est utilisé correctement ou non. On n'a pas dans le cas présent de critère de correction parce que la sensation est dénuée de tout accompagnement publiquement observable ( $S$ n'a aucune manifestation naturelle, elle n'a ni cause ni effet observable pour autrui (cf. PU 256l) et c'est pourquoi quelqu'un d'autre n'est pas en mesure de savoir quand le locuteur la ressent ni determiner quand son usage de « $S$ » est correct ou ne l'est pas. Bref, sans critère de correction, il n'y a pas de distinction (objective) entre usages corrects et usages incorrects de « $S$ » et le signe ne réfère pas à quoi que ce soit : il ne joue pas le rôle d'un authentique nom de $S$. 
Wittgenstein avance apparemment d'autres considérations à l'appui de cette conclusion ( $c f$. en particulier PU 265), mais Kripke pourrait soutenir que l'argument principal des Recherches contre l'idée d'un langage privé (qu'on trouve en particulier dans le paragraphe 258) est un corollaire de la solution sceptique. Cependant la difficulté, je pense, est la suivante. Wittgenstein fait sans aucun doute une distinction entre un langage privé et ce qu'on pourrait appeler un langage « personnel », c'est-à-dire un langage parlé par un seul individu mais qui est susceptible éventuellement d'être compris et utilisé par d'autres locuteurs. Il fait très probablement la distinction parce qu'il ne nie pas la possibilité d'un mot inventé par quelqu'un pour son propre usage, un mot que celuici pourrait partager avec d'autres locuteurs (s'il le décidait, si les circonstances étaient différentes, etc.). Il semble que l'interprétation de Kripke ait la conséquence contraire : une distinction objective usage correct / usage incorrect suppose l'existence d'une communauté d'individus ayant en commun le mot ou la règle - d'où l'impossibilité d'un langage «personnel $»^{12}$.

Je voudrais suggérer une autre lecture de l'argument et une interprétation différente de la solution sceptique qui, je pense, n'entraîne pas cette conséquence.

Que veut dire Wittgenstein par un « critère de correction »? Un critère de correction, apparemment pour Kripke, est un critère commun

12. Kripke écrit : ce que revient à nier l'argument du langage privé « est ce que l'on pourrait appeler le "modèle privé" de ce qu'est suivre une règle, lc'est-à-dire l'opinion d'après laquellel la notion qu'une personne suit une règle donnée pourrait être analysée simplement en termes de faits relatifs à celui qui suit la règle et à lui seul, sans référence à l'appartenance à une communauté plus large. » (WRPL. p. 1og) On ne peut donc pas dire de quelqu'un qu'il suit (correctement ou non) des instructions pour faire des additions s'il n'est pas membre d'un groupe dont les individus font des additions en suivant les mêmes instructions. La conséquence en est l'impossibilité d'une règle « personnelle » ou d'un mot inventé. Pourtant, Kripke écrit ensuite : « Est-ce que cela veut dire que Robinson Crusoé, isolé sur une île déserte, ne suit aucune règle, peu importe ce qu'il fait? Je ne vois pas que cela s'ensuive. Ce qui s'ensuit, c'est que si nous le concevons comme suivant des règles, nous l'introduisons Ide ce faitl dans notre communauté et lui appliquons nos critères pour dire si quelqu'un suit des règles. » (p. ıo) Mais il me semble que si quelqu'un ne peut suivre « une règle donnée » qu'à la condition que d'autres individus d'un groupe dont il est membre la suivent. comme le pense Kripke, on peut difficilement éviter la conclusion que Robinson ne suit aucune règle. Ses règles satisfont peut-être, comme le dit Kripke, « nos critères Igénéraux] pour dire si quelqu'un suit des règles », mais une telle condition est beaucoup plus faible que celle requise par la solution sceptique (cf. WRPL p. 86 à 95 pour son exposé de la solution sceptiquel. 
pour dire si un signe est employé correctement (étant commun à plusieurs locuteurs, il permet de faire une distinction objective usage correct / usage incorrect). Mais est-ce cela que veut dire Wittgenstein ? Un critère de correction pourrait être un moyen indépendant de contrôler l'usage d'un signe sans être un critère commun ou interpersonnel de correction. On pourrait imaginer la situation suivante : l'utilisateur de « $S$ » se rend compte que sa sensation est régulièrement accompagnée d'un certain phénomène observable (par exemple une élévation de sa tension artérielle [cf. PU 270] - ce que, supposons, il peut vérifier luimême à l'aide d'un manomètrel. Il dispose donc d'un critère « indépendant » pour dire s'il a la sensation (s'il choisit de considérer l'élévation de sa tension artérielle comme un critère de la récurrence de sa sensation) et, par conséquent, d'un critère pour vérifier son emploi du signe. « $\mathrm{S}$ » est maintenant un mot « personnel »; il n'est plus privé dans la mesure où le locuteur peut montrer à quelqu'un d'autre comment l'employer (ou bien il peut enseigner à autrui comment décider si son propre usage est correct ou non) et le locuteur peut dire s'il l'emploie lui-même correctement ou non (il pourrait lui arriver de penser : « Je croyais ressentir $S$ mais ce n'était pas le cas parce que le manomètre n'indique aucune élévation de ma tension artérielle »). On peut comprendre dans cette interprétation (que je ne développerai pas ici) en quel sens l'argument montre l'impossibilité d'un langage « logiquement » privé, dans lequel il n'existe aucun critère pour contrôler l'usage d'un mot, sans qu'il s'ensuive l'impossibilité d'un mot personnel - pour lequel, suivant l'argument, il doit exister un critère indépendant (personnel ou interpersonnel) de correction.

Si cette interprétation est plausible (je pense qu'elle est plus plausible que l'interprétation de l'argument, d'ailleurs courante, adoptée ici par Kripke), il devrait être possible de donner une autre interprétation, également non « communautaire », de la solution sceptique.

Pour résoudre la difficulté, on peut modifier l'interprétation de Kripke comme suit. On doit faire d'abord la distinction entre des mots (ou règles) communes et des mots « personnels ». Si inventer un mot est possible, il doit également ici y avoir une distinction (objective) correct / incorrect. Pour les mots communs, un usage correct est l'usage que la majorité des utilisateurs compétents du mot considère comme correct (la distinction est alors « objective » au sens que j'ai expliqué plus haut). D'un autre côté, supposons que « $M$ » est un exemple de mot inventé et que $R$ est la règle suivie par le locuteur quand il l'emploie (il a lui-même décidé que $R$ serait la règle qui régit son application de « $\mathrm{M} \gg$ ). Supposons aussi qu'il n'interprète pas $R$ en recourant à une autre règle. Lorsqu'il emploie $« M$ M il commet parfois des erreurs : dans 
certains cas, il l'utilise en contrevenant à la règle. En quel sens y a-t-il une distinction objective usages corrects / usages incorrects? « $\mathrm{M} »$ n'a pas d'usages intrinséquement corrects, mais il semble que la distinction soit objective en ce sens : les usages incorrects de « $\mathrm{M} »$ vont à l'encontre de $R$ telle que l'avait fixée initialement le locuteur en la comprenant comme il la comprenait à ce moment-là et sans l'interpréter à l'aide d'une autre règle (ce qui se manifeste, comme dans les autres cas, par l'« usage constant » qu'il fait de « $\mathrm{M} \gg)$, tandis que ses emplois corrects s'accordent au contraire avec ce que prescrit $R$. Autrement dit, le locuteur peut croire faire un usage correct de « $M$ » alors que ce n'est pas le cas en ce sens qu'il ne suit pas $R$ telle qu'il l'avait lui-même fixée (telle qu'il la comprenait au départ), bien que, dans certains cas, personne d'autre ne soit en mesure de dire s'il commet ou non une erreur.

On ne trouve pas à ma connaissance de textes qui vont dans le sens de cette interprétation mais elle semble compatible avec les textes cités à l'appui de la lecture de Kripke. Lorsque Wittgenstein dit que l'emploi correct d'un mot est l'emploi fait par la plupart de ses utilisateurs, il pourrait penser à des mots communs, non pas à des mots inventés et c'est ce qu'on dit ici : l'usage correct, quand on a affaire à un mot commun, est l'usage fait par la majorité de ses utilisateurs. Par ailleurs, en soutenant que l'application correcte d'une règle est l'« usage constant » (le staendiger Gebrauch) ou la «coutume » consistant à suivre la règle, il peut également avoir à l'esprit la pratique constante d'un seul individu (celle de l'auteur de la règle). Les autres textes qu'on pourrait citer en faveur de l'interprétation de Kripke semblent compatibles avec cette autre interprétation.

Pour conclure, je pense que, compte tenu de ce qui précède, on peut décrire comme suit le plan d'ensemble des Recherches, les grandes lignes de l'argumentation des Recherches des paragraphes i à 242 len excluant les remarques sur la question du langage privé) :

I) Wittgenstein avance d'abord deux de ses thèses centrales : (a) que la signification d'un mot est son usage et (b) qu'il existe une multitude d'usages des mots dans un langage (PU I-26). Les arguments à l'appui de ces deux thèses sont surtout indirects (il fait en particulier la critique de la conception du langage du Tractatus). (II) Les paragraphes suivants

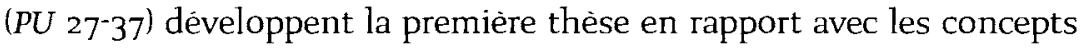
de nomination et de définition ostensive : un nom ne dénote quelque chose qu'à la condition qu'il ait un usage; une définition ostensive n'explique la signification d'un nom que si l'usage ou le rôle du nom dans le langage a déjà été fixé. (III) Les paragraphes qui suivent (PU 38-64) traitent du concept de nomination (lequel a une importance particulière dans le Tractatus et dans l'atomisme logique de Russell) : Wittgenstein fait la 
critique de l'opinion selon laquelle le mot « ceci » est un nom (le seul authentique nom pour Russell) et du point de vue du Tractatus qui veut que les mots figurant dans les propositions élémentaires complètement « analysées » dénomment des objets « simples ». (Iv) Les paragraphes 65 à 88 ont un rôle de transition. Wittgenstein pose maintenant la question: $y$ a-t-il quelque chose de commun à tous les jeux de langage (ou à tous les usages du langage) ? La réponse, que suggère l'ensemble des remarques qui précèdent, est qu'ils n'ont rien (en tout cas rien $\mathrm{d}^{\prime}$ « important ») en commun : on ne trouve entre ces différents usages que des « ressemblances de famille». En développant cette réponse, à l'aide de l'exemple du mot « jeu», Wittgenstein passe à une autre question : notre usage du langage est-il partout circonscrit par des règles ? Existe-t-il des règles strictes de langage? Sa réponse est négative et la conséquence qui s'ensuit est qu'il n'y a rien de tel qu'un usage correct des mots dans un langage. (v) Les paragraphes 89 à i37 sont une digression : Wittgenstein compare et oppose son nouveau projet philosophique des Recherches à celui du Tractatus (il fait entre autres des remarques critiques sur la conception des règles du Tractatus). (vi) Dans les paragraphes 138 à 184 , il soutient (ce qu'on peut considérer comme une autre de ses thèses centrales) qu'il n'existe aucun lien conceptuel entre, d'un côté, les concepts de signification ou de compréhension (donc d'usage) et, de l'autre, des concepts psychologiques : la compréhension d'un mot ne consiste pas dans un état dispositionnel (une structure) de l'esprit des locuteurs ni dans des « expériences conscientes » telles qu'avoir présente à l'esprit une image mentale, une « idée générale », etc.. (vir) Enfin, dans les paragraphes 185 à 242 - la seconde série importante de remarques sur les règles - il fait la critique de la solution « directe » du paradoxe d'après laquelle la source de l'usage correct des mots est leur «signification » (comprise au sens de quelque chose qui a son siege dans l'esprit des locuteurs) et il suggère une solution «sceptique »: la correction de l'emploi des mots dans un langage se manifeste dans - et n'est rien d'autre que - l'« usage constant » qu'en font ceux qui l'utilisent ${ }^{\mathrm{I}}$.

\section{Département de philosophie Cégep de Saint-Hyacinthe}

13. Les recherches qui ont mené à la rédaction de cet article ont été subventionnées par le FCAR. 